in multivariate analysis (age, sex, LVH, septum T1, T2 in the BIFL, GLS, LGE) was T2 in the BIFL wall $(\beta=0.4, p=0.001)$.

Conclusions Cardiac involvement in FD goes beyond storage (low T1 values). When LGE is present, this is almost always associated with a high T2 and troponin elevation supporting FD as a chronic inflammatory cardiomyopathy. Initial reports of LGE being fibrosis are too simplistic - LGE in FD appears to have a significant chronic inflammation/oedema component.

\section{NEO-AORTIC DILATATION AND REGURGITATION DURING PREGNANCY FOLLOWING THE ROSS PROCEDURE: AN EVALUATION OF CARDIAC MAGNETIC RESONANCE (CMR) DATA}

V Stoll, L Hudmsith, P Clift. University of Birmingham, Adult Congenital Heart Disease Unit, Queen Elizabeth Hospital, Birmingham, UK

\subsection{6/heartjnl-2019-BSCMR.2}

Background The neo-aorta produced by the pulmonary autograft following the Ross procedure has excellent long-term outcomes, with an increasing number of women now undergoing pregnancy following Ross. Cardiac Magnetic Resonance (CMR) has been used to evaluate the risk of progression of neo-aortic dilatation and dissection that may arise as a result of the additional cardiovascular risks posed during pregnancy. Although there have been reported cases of dilatation and dissection, there is little research.

Aims To evaluate the extent of neo-aortic dilatation, and associated aortic regurgitation, during pregnancy following the Ross procedure.

Methods A retrospective cohort of women were identified who had undergone a Ross procedure after 1985, who in January 2019 were aged $>16$, in an adult congenital cardiology and joint cardiac-obstetric centre. CMR data was used to evaluate neo-aortic root dimensions and aortic regurgitation extent pre-, during and post- pregnancy. A control group of casematched patients who had undergone the Ross procedure, but not pregnancy, was used for comparison.

Results In all, 8 women carried a total of 15 pregnancies to term. In only one pregnancy did the mother experience serious cardiac decompensation, necessitating early delivery and associated with subaortic stenosis, mild aortic root dilatation and increasing aortic regurgitation. Four women (50\%) experienced an element of aortic root dilatation during pregnancy, with an average increase between them of $0.525 \mathrm{~cm}$ (range +0.4 to $+0.7)$. In no case did this lead to progressive dilatation or dissection. When compared to the control group, pregnancy was found to carry an increased risk of neo-aortic dilatation (Mean aortic root change for group: $+0.26 \mathrm{~cm}$ (SD 0.30) pregnancy vs. $+0.16 \mathrm{~cm}$ (SD 0.27) control). There was a high rate of initial mild aortic regurgitation in both groups. In three women their extent of regurgitation increased post-pregnancy, correlated with increased parity and aortic dilatation. In the control group only two women experienced increased regurgitation, only one of which was associated with neo-aortic dilatation. Conclusions Pregnancy appears generally well tolerated by women following the Ross Procedure, although there is an increased risk of neo-aortic dilatation and corresponding increase in aortic regurgitation compared to the control group.

\section{TRAIN THE AI LIKE A HUMAN OBSERVER: DEEP LEARNING WITH VISUALISATION AND GUIDANCE ON ATTENTION IN CARDIAC T1 MAPPING}

Qiang Zhang, Konrad Werys, Elena Lukaschuk, Iulia Popescu, Evan Hann, Stefan Neubauer, Vanessa M Ferreira, Stefan K Piechnik. OCMR, University of Oxford Centre for Clinical Magnetic Resonance Research

\subsection{6/heartjnl-2019-BSCMR.3}

Background Artificial intelligence (AI) is increasingly used in diagnostic imaging. Deep convolutional neural networks (CNN)

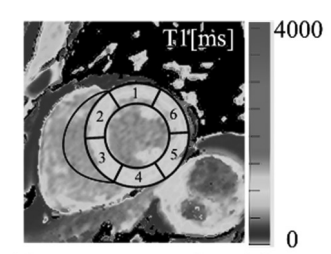

(a) AHA segmental model

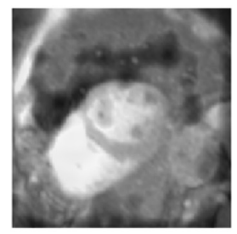

(b) Attention of an ill-trained CNN

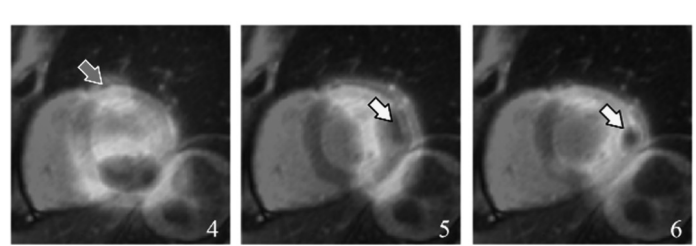

(c) Attention of a well-trained $\mathrm{CNN}$ when detecting artefacts in 6 segments
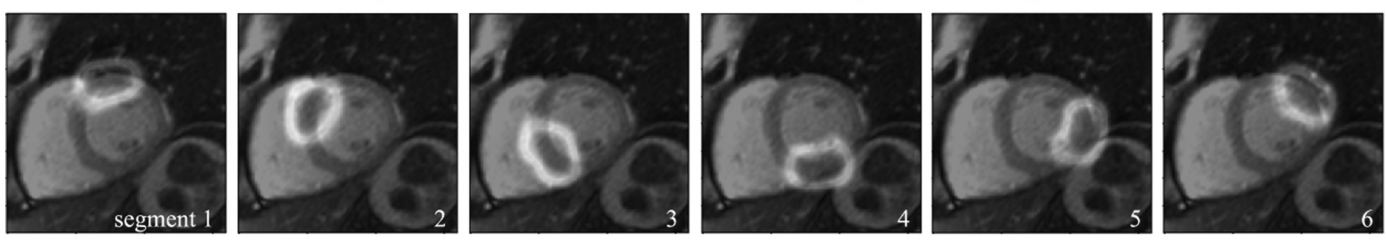

(d) $\mathrm{CNN}$ trained with proposed attention guidence technique

Abstract 3 Figure 1 Attention maps as the CNN 'eye tracker' in detecting T1-mapping artefacts in the 6 AHA segments (a), which reveals that (b) an ill-trained CNN looked at the features not desired for the task; In comparison; (c) a well-trained CNN correctly identified the segments, but with distraction (red arrows) and low accuracy (yellow arrows); (d) CNN trained with attention guidance looked at the target myocardial segments more accurately 
can learn from labelled datasets, and then provide independent interpretations on new cases, but often without traceability to how such conclusions were made. This 'black box' behaviour is not desirable for clinical applications. We propose a novel framework for visualising and guiding the AI attention, using artefact detection in cardiac T1-mapping as an example - a critical quality assurance step for clinically-robust T1 determinations.

Method We utilised an AI attention visualisation framework. This serves as an 'eye tracker' and reveals where the neural network 'looks' when scoring artefacts. The technique adds an essential accountability aspect to the $\mathrm{CNN}$ by producing additional evidence to validate the decision-making process. Beyond simply observing the AI attention maps, we provided additional direct guidance on the attention of the CNN, instructing the machine where to look, similar to training a human operator. Results We demonstrate an application in automated T1-mapping artefact detection of the 6 AHA segments in mid-ventricular slices (figure 1a). The AI 'eye tracker' detected an illtrained CNN not paying attention to myocardium (figure $1 \mathrm{~b}$ ). A well-trained CNN learned from the training data to pay attention to the 6 myocardial segments, but with distraction by other image features (red arrows, figure 1c) and inaccuracy (yellow arrows). The proposed solution is a CNN trained with additional guidance to pay attention to the correct structures and avoid distractions (figure 1d).

Conclusion CNN designed with both visualisation in perception and guidance on attention to relevant anatomical structures can lead to significantly more transparent and accountable AI, therefore more reliable for clinical practice.

\section{MANGANESE ENHANCED T1 MAPPING OF CALCIUM HOMEOSTASIS IN ACUTE MYOCARDIAL INFARCTION}

${ }^{1,2} \mathrm{NH}$ Jasmin, ${ }^{1} \mathrm{M}$ Zaw-Thin, ${ }^{1} \mathrm{MF}$ Lythgoe, ${ }^{3} \mathrm{~S}$ Davidson, ${ }^{1} \mathrm{DJ}$ Stuckey. ${ }^{1}$ University College London, Centre for Advanced Biomedical Imaging, London, UK; ${ }^{2}$ Universiti Sultan Zainal Abidin, School of Medical Imaging, Terengganu Darul Iman, Malaysia; ${ }^{3}$ University College London, Hatter Institute, London, UK

10.1136/heartjnl-2019-BSCMR.4
Introduction Manganese is a calcium analogue and potent T1contrast agent for MRI that enters cardiomyocytes through active calcium channels, thus reducing T1 in viable myocardium. ${ }^{1}$ We hypothesized that T1-mapping-MRI could measure $\mathrm{Mn}$ levels in the myocardium acutely after myocardial infarction (MI) allowing quantification of intracellular $\mathrm{Ca}$ response to ischemic injury.

Methods Mice received intraperitoneal injections of $0.10 \mathrm{mmol} / \mathrm{kg} \mathrm{MnCl}_{2} 40 \mathrm{~min}$ before MI. T1-mapping-MRI was performed at 1, 2 and 3 hours and 2 days after MI. R1 values $(1 / \mathrm{T} 1=$ the relaxivity of the tissue) were analysed from the area-at-risk of infarction segments (AAR-MI, $n=12$ ) and viable segments (Viable-MI, $\mathrm{n}=12$ ) of infarcted hearts, and naïve control heart (Viable-Naïve, $\mathrm{n}=12$ ). Imaging was performed using a 9.4T Agilent MRI system and a multi inversion time Look-Locker sequence in the short-axis orientation (TE/

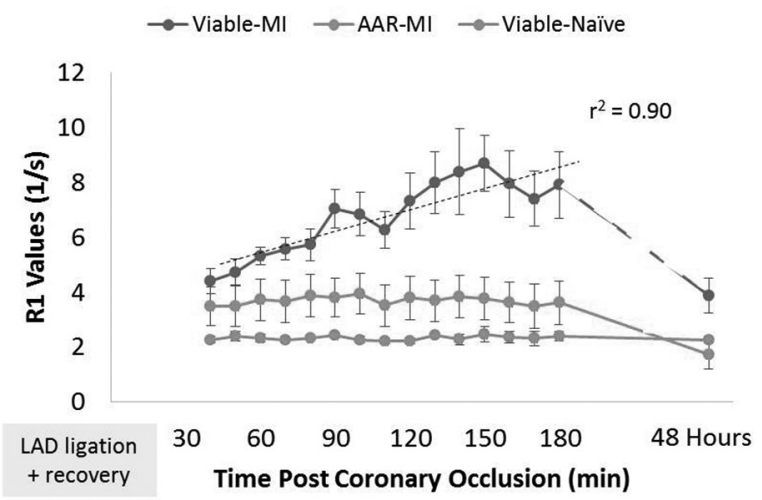

Abstract 4 Figure 2 R1 values in acute myocardial infarction after Mn infusion

$\mathrm{R} 1$ values in MI mouse hearts at 1, 2, 3 \& $48 \mathrm{~h}$ after myocardial infarction and in naïve mouse hearts at the same time points after $\mathrm{Mn}$ infusion. Viable-MI = Remote myocardium at 1 to 3 hours after occlusion, AAR-MI = Area at risk at 1 to 3 hours after occlusion, Viable-Naïve $=$ Remote myocardium in naïve control group

\section{(a) 1 Hour Post MI}

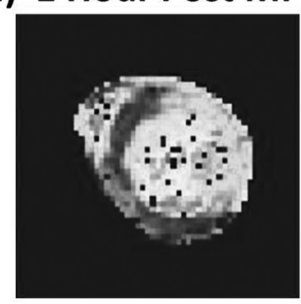

(b) 1 Hour Naïve

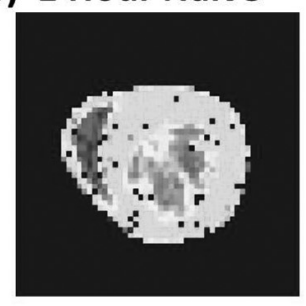

2 Hours Post MI
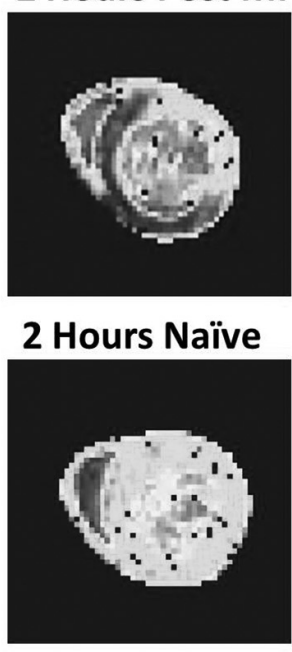

\section{Hours Post MI}
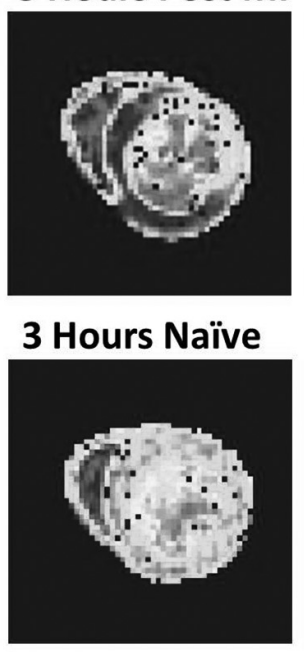
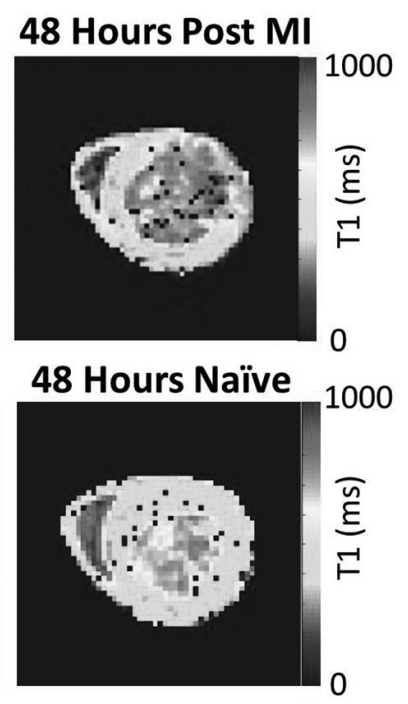

Abstract 4 Figure 1 T1 Maps of Mn uptake in acute myocardial infarction

T1 Maps acquired at 1,2,3 and $28 \mathrm{~h}$ after coronary occlusion (top) or in naïve mouse hearts (bottom). In the first hours after Ml, T1 in viable segments of infracted hearts was decreased compared with the area at risk of Ml and the naive myocardium owing to increased Mn uptake. By $48 \mathrm{~h}$, $\mathrm{T} 1$ in the infarct was higher than viable segments owing to reduced $\mathrm{Mn}$ uptake in the non-contractile ischemic zone 